\title{
HOT OFF THE PRESS: CLINICAL SGLT2 inhibition reduces blood pressure and left ventricular mass
}

\author{
THOMAS KAHAN
}

Karolinska Institutet, Department of Clinical Sciences, Danderyd Hospital, Division of Cardiovascular Medicine, \& Department of Cardiology, Danderyd University Hospital Corporation, Stockholm, Sweden

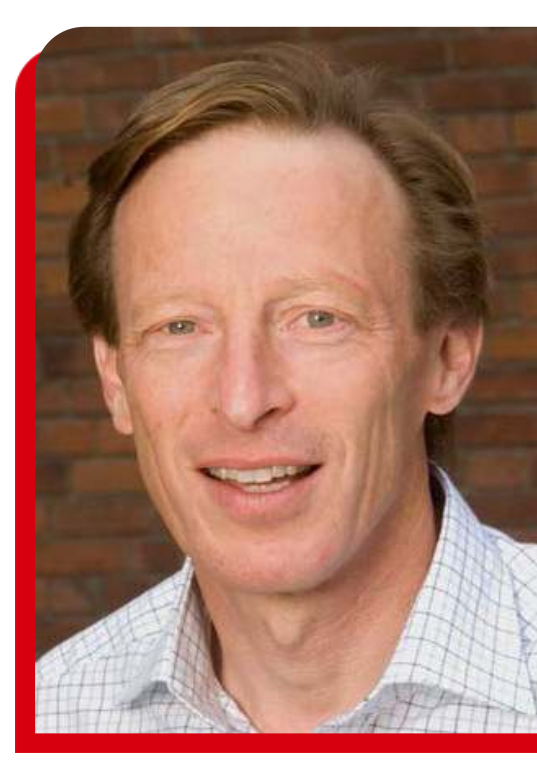

Sodium-glucose cotransporter 2 (SGLT2) inhibitors were developed to reduce blood glucose levels in patients with poorly controlled type 2 diabetes. More important, however, is their ability to reduce cardiovascular morbidity and mortality in patients with type 2 diabetes and atherosclerotic disease; and this benefit may be regardless of the presence or absence of diabetes (at least among patients with heart failure and a reduced ejection fraction). There are several proposed mechanisms by which SGLT2 inhibitors may exert their effects to reduce cardiovascular events, including improved glucose control, diuresis, vasodilatation with reductions in blood pressure, preload and afterload, and alterations in cardiomyocyte function ${ }^{1,2}$.

Recently, Verma and colleagues ${ }^{3}$ reported on the effects of the SGLT2 inhibitor empaglifozin on blood pressure and left ventricular mass in patients with type 2 diabetes and coronary artery disease. The EMPA-HEART Cardiolink- 6 trial randomized 97 patients (93\% men) to double blind treatment with $10 \mathrm{mg}$ empaglifozin od or placebo for six months; 90 patients were available for outcome evaluation. Mean age was of 63 years, duration of type 2 diabetes 11 years, glycated haemoglobin $8.0 \%$, estimated glomerular filtration rate $88 \mathrm{~mL} /$ $\mathrm{min} / 1.73 \mathrm{~m} 2$. Mean $24 \mathrm{~h}$ ambulatory blood pressure was $139 / 80$ and $138 / 88 \mathrm{~mm} \mathrm{Hg}$, and left ventricular mass index (by cardiac magnetic resonance imaging) 59 and $62 \mathrm{~g} / \mathrm{m} 2$ in the empaglifozin and the placebo group, respectively. Primary outcome of the study was the change in left ventricular mass index during six months.

Compared to placebo, empaglifozin reduced $24 \mathrm{~h}$ ambulatory systolic and diastolic blood pressure by (mean between group difference and 95\% Cl) -6.8 $[-11.2 ;-2.3] \mathrm{mm} \mathrm{Hg}(\mathrm{P}=0.003)$ and $-3.2[-5.8 ;-0.6]$ $\mathrm{mm} \mathrm{Hg}(\mathrm{P}=0.016)$. Also left ventricular mass index was reduced more by empaglifozin than by placebo, -3.4 $[-5.9 ;-0.8] \mathrm{g} / \mathrm{m} 2(P=0.01)$. The changes in glycated haemoglobin by empaglifozin and placebo were small (-0.4 and $-0.3 \%$, respectively).

This is the first randomized clinical trail to show that empaglifozin reduces LV mass. Furthermore, this study confirms findings of a reduction in ambulatory blood pressure by SGLT2 inhibitors ${ }^{4}$. The blood pressure reduction is not trivial. Of note, the reduction in left ventricular mass did not relate to changes in blood pressure, suggesting an effect beyond that of blood pressure reduction alone. A similar dissociation between effects on blood pressure and on myocardial geometry and function has also been observed when antihypertensive drug classes have been compared. Hypertensive heart disease is a strong cardiovascular risk factor, and regression of left ventricular mass reduces future cardiovascular events ${ }^{5}$. Thus, the results of the current study may help our understanding the mechanism(s) for the reduction in cardiovascular events in patients treated with SGLT2 inhibitors. 


\section{References:}

1.Verma S, McMurray JJV. SGLT2 inhibitors and mechanisms of cardiovascular benefit: a state-of-the-art review. Diabetologia 2018; 61:2108-2117. doi: 10.1007/ s00125-018-4670-7

2. Chin KL, Ofori-Asenso R, Hopper I, et al. Potential mechanisms underlying the cardiovascular benefits of sodium glucose cotransporter 2 inhibitors: a systematic review of data from preclinical studies. Cardiovasc Res 2019;115:266-276. doi: 10.1093/cvr/cvy295

3. Verma S, Mazer CD, Yan AT, et al. Effect of empagliflozin on left ventricular mass in patients with type 2 diabetes mellitus and coronary artery disease: The EMPA-HEART CardioLink- 6 randomized clinical trial. Circulation 2019;140:1693-1702. doi: 10.1161/ CIRCULATIONAHA.119.042375

4. Kario K, Okada K, Kato M, et al. 24-hour blood pressure-lowering effect of an SGLT-2 inhibitor in patients with diabetes and uncontrolled nocturnal hypertension: results from the randomized, placebo-controlled SACRA study. Circulation 2019;139:2089-2097. doi: 10.1161/CIRCULATIONAHA.118.037076

5. Jekell A, Nilsson PM, Kahan T. Treatment of hypertensive left ventricular hypertrophy. Curr Pharm Des 2018;24:4391-4396. doi: 10.2174/13816128256661 81203092918

\section{HOT OFF THE PRESS: BASIC}

Circulating Extracellular Vesicles in Normotension Restrain

\section{Vasodilation in Resistance Arteries}

\author{
DYLAN BURGER \\ Ottawa Hospital Research Institute \\ Ottawa, Canada
}

DOI: $10.30824 / 2003-6$

Extracellular vesicles (EVs) are particles naturally released from a cell that are surrounded by a lipid bilayer and lack replicative capacity ${ }^{1}$. In recent years they have received significant attention as both biomarkers and mediators of intercellular signaling. In particular, circulating populations of large extracellular vesicles (i.e. microparticles or microvesicles) are elevated in conditions of vascular injury, strongly correlated with measures of vascular health, and predictive of future adverse cardiovascular events 2. Our laboratory, and others, have also shown that large EVs can serve as vectors for intercellular communication leading to endothelial injury ${ }^{3}$.

Interestingly, while there has been considerable interest in the impact of various cardiovascular conditions on circulating EVs, the impact of hypertension has been comparatively understudied. In the January issue of Hypertension work from the laboratory of Dr. Uta Erdbrügger addresses this critical gap in knowledge ${ }^{4}$. Good et al examined levels of circulating EVs in plasma from spontaneously hypertensive rats (SHR) and normotensive Wistar Kyoto Rats (WKY). At 12 weeks of age they observed significant increases in levels of circulating large EVs arising from endothelial cells as well as leukocytes. More importantly, they provided the first functional analysis of the impact of hypertension on the bioactivity of circulating EVs.

First, they observed that large EVs isolated from normotensive rats reduced vasodilation in isolated resistance arteries. This observation was consistent with previous reports from Brodsky et al. ${ }^{5}$ and others. Most interestingly however was the observation that this impairment in vasorelaxation is absent in large EVs isolated from hypertensive rats. This was also true for EVs isolated from normotensive vs hypertensive human subjects. The authors also 